\title{
La riflessione sul romanzo nell'Europa del Settecento, a cura di Rosamaria Loretelli e Ugo M. Olivieri
}

\section{Maurizio Nascimbene}

\section{(2) OpenEdition}

1 Journals

\section{Edizione digitale}

URL: http://journals.openedition.org/studifrancesi/27102

DOI: 10.4000/studifrancesi.27102

ISSN: 2421-5856

\section{Editore}

Rosenberg \& Sellier

\section{Edizione cartacea}

Data di pubblicazione: 31 décembre 2006

Paginazione: $564-565$

ISSN: 0039-2944

\section{Notizia bibliografica digitale}

Maurizio Nascimbene, "La riflessione sul romanzo nell'Europa del Settecento, a cura di Rosamaria Loretelli e Ugo M. Olivieri », Studi Francesi [Online], 150 (L | III) | 2006, online dal 30 novembre 2015, consultato il 08 novembre 2020. URL : http://journals.openedition.org/studifrancesi/27102 ; DOI : https://doi.org/10.4000/studifrancesi.27102

\section{(c) (i) $\odot$}

Studi Francesi è distribuita con Licenza Creative Commons Attribuzione - Non commerciale - Non opere derivate 4.0 Internazionale. 
AA. VV., La riflessione sul romanzo nell'Europa del Settecento, a cura di Rosamaria Loretelli e Ugo M. Olivieri, Milano, Franco Angeli, 2005, pp. 164.

Come apprendiamo dall'Introduzione (pp. 7-9) a cura di OLIVIERI, gli studi sul romanzo del Settecento mostrano che accanto all'apparizione «della nuova forma narrativa del novel» il XVIII secolo ha generato tutta «una produzione saggistica dedicata a discutere e analizzare $i$ tratti innovativi del genere». Data l'importanza di tali discussioni teoriche sempre più la critica moderna ha partorito ricerche finalizzate a gettare nuova luce sui romanzi di quel periodo, e in tale ottica si situa anche il convegno dal titolo «La riflessione sul romanzo nell'Europa del Settecento» organizzato a Napoli il 23 e il 24 ottobre 2003 dalla Società Italiana di Studi sul secolo XVIII, dall'Istituto Italiano per gli Studi Filosofici e dal Dipartimento di Filologia Moderna "Salvatore Battaglia" dell'Università partenopea "Federico II". Il presente volume a cura di Rosamaria Loretelli e Ugo Olivieri raccoglie gli atti di queste giornate di dibattito accademico nell'intento di fornire agli studiosi un utile compendio di interventi finalizzati soprattutto a indagare il ruolo dell'Italia all'interno di una più prolifica dimensione franco-inglese.

In questo contesto, Giorgio Cusatelli (Intorno al romanzo settecentesco, pp. 11-16) inquadra le linee generali dell'argomento oggetto di studio - vale a dire il romanzo settecentesco - prestando attenzione alla «dimensione sociologica» della ricerca. In tale ambito, Cusatelli rileva quanto importante fosse la fruizione da parte del pubblico femminile e quanto significativi fossero la produzione (all'interno della quale elenca le ripartizioni del genere messe in evidenza da Alain Montandon) e il consumo del romanzo in nazioni quali la Francia e l'Inghilterra, specie se a tutto ciò si paragona la scarsità e la mediocrità del prodotto italiano.

Rosamaria LORETELLI (Dell'unità narrativa, ovvero la riflessione settecentesca inglese sul roman$z o$, pp. 17-38) approfondisce il divario esistente tra la produzione romanzesca franco-inglese e quella italiana, e scopre che la maggior fortuna della nuova forma narrativa in terra britannica è dovuta soprattutto all'intenso dibattito creatosi intorno a quel prodotto che «tutti da un certo momento in poi non hanno più esitato a chiamare novel». Fornendo puntuali spiegazioni, supportate da citazioni d'autore (Fielding, Congreve, Smollett,...), sulla riflessione legata al nome più consono da adottare per il nuovo genere in sviluppo, nonché dei suoi stessi contenuti e strutture formali, Loretelli ci restituisce l'intensità di un dibattito «reader-oriented» che, svolgendosi nel cuore di «prefazioni e commenti interni ai romanzi, lettere e diari, articoli di giornale e an- che recensioni», è riuscito a far breccia sui lettori gettando, così, le premesse capaci di porre fine al «labirinto degli sperimentalismi» a vantaggio di quel novel che «si consoliderà nel grande romanzo ottocentesco».

Annamaria LAMARRA (La teoria del romanzo nel novel of manners di Frances Burney, pp. 3947), invece, concentra la propria attenzione sulla differenza sostanziale tra la narrativa del periodo precedente, in cui «il potere dell'irreale [...] alimentava gli universi fantasmatici del gotico», e quella emergente durante il secolo dei Lumi, in cui si «privilegia come filo conduttore della trama "i modi e gli atteggiamenti tipici dei tempi", cui guardano con crescente interesse i nuovi soggetti sociali». Al centro dell'indagine della studiosa v'è il novel of manners di Frances Burney, che pone quale cardine del proprio operare la più concreta aderenza del narrato alla natura dalla quale $\mathrm{i}$ personaggi sono ricavati, in modo tale da fornire «"l'immagine di una supposta, e tuttavia naturale e possibile esistenza umana"».

Mirella BRINI SAVORELLI ( $A$ maze, but not without a plan. Di una linea di pensiero a proposito del narrare in Francia nel Settecento, pp. 49-59) si mostra affascinata da un tema che, tra i testi da lei presi in esame, ritrova anche in una pagina di Isabella Biagini: il metaromanzo. Partendo da una citazione dalle Lettres persanes di Montesquieu, Brini Savorelli analizza le digressioni interne ai romanzi settecenteschi, mettendone in luce eventuali positività e negatività nell'ottica della leggibilità del prodotto e della sua capacità di conversare con i lettori.

Pino Fasano (Il romanzo inesistente, pp. 61-75) ricerca le motivazioni per cui l'Italia settecentesca è considerata un po' il fanalino di coda europeo per quanto riguarda la produzione e la commercializzazione del nuovo romanzo. Ancor più, $\mathrm{Fa}$ sano si spinge a sottolineare come si sia persino giunti a negare l'esistenza di un romanzo italiano settecentesco, mettendo in luce come i suoi protagonisti (vedasi un Pietro Chiari), a differenza di quanto accadeva nel dibattito francese o inglese, tendessero addirittura «a svilire la rilevanza della [loro stessa] vocazione narrativa». Dopo una lucida e puntuale analisi, Fasano conclude accettando parzialmente l'ipotesi dell'inesistenza del romanzo italiano settecentesco semplicemente spostando gli accenti del giudizio: egli, infatti, seguendo l'ideologia di Friedrich Schlegel, ritiene che se si considerasse il roman come «il necessario elemento di ogni forma poetica», cosicché «un romanzo» non sarebbe altro che «un libro romantico», si renderebbe pienamente conto del- 
la mancanza di tale roman, «come genere stretto», nell'Italia del XVIII secolo.

Carlo Alberto MadRIGNANI (Il romanzo, catechismo per le riforme, pp. 77-101), prestando la propria attenzione alla napoletana Società letteraria e tipografica diretta dal «padrone culturale» Giuseppe Maria Galanti, «scolaro dichiarato di Genovesi», torna a sua volta a quella che può essere definita la dimensione sociologica del romanzo durante il secolo preso in esame. In modo particolare, Madrignani vede nel romanzo il «centro di quella rivoluzione del libro» inauguratasi «da quando un'editoria popolare ed effimera [è riuscita] a conquistare zone di pubblico che sembrano emergere dal nulla». Questo «genere selvaggio», «refrattario agli statuti sia della "buona letteratura" che del vivere civile», rendeva conto di una cultura «bassa», che imparava a educarsi sulle pagine avventurose e passionali del nuovo prodotto, parallela e contrapposta a una «alta» ufficiale e di Chiesa. Anche per questo, a suo avviso, «nessun potente se ne [fece] protettore», e anche per questo l'Italia si trovò arretrata rispetto alle culture estere di Francia e Inghilterra.

Daniela MangIone (Ruoli e funzioni di autore e lettore nel dibattito settecentesco italiano sul romanzo, pp. 103-117) ritiene che l'inglese «contrapposizione fra Story ed Hystory», che ruota attorno al concetto di «verità-menzogna», contenga in sé una sfumatura che «sembra mancare nel mondo francese e nel nostro». Rimaneva, tuttavia, in Italia una rigida «distinzione concettuale tra storiografia e narrazione». Consci di tutto ciò, pur mancando nel settecento della Penisola un vero e proprio dibattito sul romanzo diffuso presso il grande pubblico, gli autori prestavano una certa attenzione verso il «lettore non colto» e, per tale motivo, seminavano nei relativi «interventi di riflessione sul romanzo» le loro «preoccupazioni morali circa gli effetti degli esempi romanzeschi sugli individui» in un modo tale che «l'incauto lettore» veniva guardato pressappoco nella stessa maniera con la quale la Chiesa considerava i propri discepoli.

Matteo A. Palumbo (Foscolo e il romanzo: riflessioni intorno a un genere letterario, pp. 119-128) si concentra soprattutto sulle riflessioni foscoliane intorno al nuovo genere letterario che emergono da testi quali il Saggio di novelle di Luigi Sanvitale, il Saggio sulla letteratura italiana contemporanea e le Ultime lettere di Jacopo Ortis. Come si può intuire dai suddetti titoli, «Foscolo collega all'attività più propriamente creativa una sistemazione critica» nell'intento d'inserire il romanzo «nella costellazione delle forme e dei linguaggi letterari», riconoscendo in esso le doti, tra le quali spicca soprattutto la «funzione di mimesi storica», in grado di distinguerlo dalla poesia. Questa fedeltà alla storia, però, non deve essere «una tendenziale archeologia del passato», ma un'oscillazione «di un'anima, esposta alla disarmonia della storia». I romanzi, perciò, devono «trasmettere ai lettori l'immagine di "una realtà che riscuote profondo interesse negli Italiani, e ha pure la forza di colpire l'attenzione dei lettori stranieri" ». Di Walter Scott, allora, si possono riprendere metodi e fini, $\mathrm{ma}$ - attraverso l'uso della forma epistolare, più adatta allo scopo perseguito - «descrivere "i contemporanei e i viventi"».

Clotilde BERTONI (Il romanzo in discussione: il discorso metanarrativo nel Fermo e Lucia, pp. 129-144) presta attenzione alle differenze esistenti tra i manzoniani Fermo e Lucia e I Promessi Sposi. In modo particolare, l'interesse della Bertoni è veicolato verso l'analisi dell' «intrusione massiccia del narratore» - così consistente da creare «pause discorsive tali da incrinare i confini fra narrativa e saggio» - che va sempre più riducendosi man mano che prende forma la versione definitiva del capolavoro dato alle stampe. La metanarratività dei Promessi Sposi è «meno aggressiva e meno vulnerabile», pur mantenendo sempre «saldamente il monopolio del discorso», come prova la «gestione della digressione, sempre accentrata nelle [...] mani» di Manzoni. Il lavoro dell'autore, come rileva la Bertoni, corrisponde a un preciso intento: coinvolgere il lettore e stimolarlo. Manzoni, però, comprese l'importanza di abbassare un poco la propria voce, di dissimulare le proprie riflessioni, affinché il suo lettore si lasciasse «dominare dalla morale palese dell'opera» o si addentrasse «nei suoi significati riposti», ma lo facesse in piena libertà d'agire.

Grazia MeLli (Appunti sul Manzoni. La tragedia, il romanzo e la morale cattolica, pp. 145-161) torna anch'ella a Manzoni e alle sue riflessioni intorno al romanzo. La studiosa cita soprattutto quanto emerge di quelle meditazioni dalle varie lettere, e riporta nuovamente alla ribalta il problema «del rapporto fra storia e invenzione» che spinge l'autore a confrontarsi necessariamente con «lo scoglio del romanzesco» (definizione attribuita da Manzoni «al romanzo francese del Seicento») nell'intento di fondare un «modello alternativo, aderente al vero che la storia propone, e integrativo dei vuoti che essa non colma». Manzoni, nelle lettere, tratta anche della tragedia e non manca di puntualizzare la propria opinione anche riguardo al «ruolo formativo e pedagogico dell'intellettuale» (argomento che riprenderà poi «nella prefazione al Conte di Carmagnola»), discorso in cui emerge pure la necessità di rinnovare e attualizzare, tramite un «fruttuoso confronto con le istanze civili che ispirano il dibattito letterario contemporaneo», «le premesse della morale cattolica».

[MAURIZIO NASCIMBENE] 